\title{
Das Millenniumsproblem der Navier-Stokes-Gleichungen
}

\author{
Reinhard Farwig
}

\begin{abstract}
Eines der sieben Millenniumsprobleme des Clay Mathematics Institute betrifft die Regularität von Lösungen der instationären Navier-Stokes-Gleichungen in drei Raumdimensionen. Eine offizielle Beschreibung des Problems im Ganzraum und im periodischen Fall $(\mathbb{R} /(2 \pi \mathbb{Z}))^{3}$ findet man bei Ch. Fefferman $([18])$. In diesem Artikel werden wir die Navier-Stokes-Gleichungen einführen, ihre wesentlichen mathematischen Schwierigkeiten und Lösungsansätze beschreiben, beginnend mit der grundlegenden Arbeit von J. Leray (1934), und zu neuesten Ergebnissen über Nicht-Eindeutigkeit und Beispielen mit Singularitäten kommen. Auf dieser tour de force werden wir das offene Regularitätsproblem erklären.
\end{abstract}

Die Navier-Stokes-Gleichungen sind das Standardmodell zur Beschreibung viskoser, inkompressibler Flüssigkeiten wie z. B. Wasser; dagegen sind Modelle für polymere Fluide, Blut oder Öle komplizierter und beinhalten weitere nichtlineare Terme. Die klassischen Erhaltungsgesetze für Masse und Impuls liefern für das Geschwindigkeitsfeld $u=\left(u_{1}, u_{2}, u_{3}\right)$ und den hydrostatischen Druck $p$ das nichtlineare partielle Differentialgleichungssystem

$$
\begin{array}{r}
u_{t}+u \cdot \nabla u-v \Delta u+\nabla p=f \\
\operatorname{div} u=0
\end{array}
$$

Der einzige nichtlineare Term $u \cdot \nabla u$ steht für $\sum_{j=1}^{3} u_{j} \frac{\partial u}{\partial x_{j}}$ und entsteht auf natürliche Weise als totale Zeitableitung der Geschwindigkeit $u(x(t), t)=\dot{x}(t)$ entlang der Trajektorie $x(t)$ eines Partikels, d.h. als die Beschleunigung

$$
\begin{aligned}
a(x(t), t) & =\frac{\mathrm{d}}{\mathrm{d} t} u(x(t), t) \\
& =\sum_{j=1}^{3} \dot{x}_{j}(t) \frac{\partial u}{\partial x_{j}}+\frac{\partial u}{\partial t} .
\end{aligned}
$$

Die Bedingung div $u=0$ impliziert mit dem Gaußschen Satz die Inkompressibilität des Fluids. Aufgrund des Newtonschen Gesetzes zur Viskosität und der Stokesschen Hypothese (1845), die im Wesentlichen schon 1843 von A. Barré de Saint-Venant benutzt wurde, wird die Viskosität durch den Term $-v \Delta u$ mit dem Koeffizienten $v>0$ modelliert. Der Einfachheit halber sei die konstante Dichte $\rho=1$. Das System (1), (2) wird geschlossen durch einen Anfangswert $u_{0}$ für die Geschwindigkeit zur Zeit $t=0$ und Bedingungen an $u$ auf dem Rand $\partial \Omega$ des Gebietes $\Omega \subset \mathbb{R}^{3}$, es sei denn $\Omega=\mathbb{R}^{3}$ oder $\Omega$ ist der Torus $\mathbb{T}^{3}$ mit periodischen Randbedingungen. Die übliche Randbedingung ist die noslip-Bedingung $u=0$ auf $\partial \Omega$, d. h., Partikel auf $\partial \Omega$ zur Zeit $t=0$ bleiben für alle Zeiten am Rand haften.

\section{Grundlegende Beobachtungen}

Die Navier-Stokes-Gleichungen (1)-(2) sind ein gekoppeltes System nichtlinearer partieller Differentialgleichungen, so dass wie üblich die Frage nach zeitlich globalen Lösungen und Eindeutigkeit auftritt. Allerdings greifen klassische Techniken wie z. B. das Maximumprinzip beim Poisson-Problem $-\Delta u=f$ oder der Wärmeleitungsgleichung $u_{t}-\Delta u=f$ für das Geschwindigkeitsfeld $u$ nicht: Bei gegebenen Randdaten $u \neq 0$ auf $\partial \Omega$ kann z. B. im stationären Fall die a priori-Abschätzung

$$
\max _{x \in \Omega}|u(x)| \leq \max _{y \in \partial \Omega}|u(y)|
$$

nicht (!) erwartet werden. Als Beispiel betrachte man eine Strömung in einer endlichen Röhre mit einer Verjüngung zwischen dem Ein- und Ausströmrand. Dann wird $|u|$ aufgrund der Inkompressibilität, div $u=0$, das Maximum in der Engstelle und nicht am Rand annehmen.

Der Druck hat zwar eine große physikalische Bedeutung, führt aber auch zu erheblichen mathematischen Schwierigkeiten. Für die stationären Stokes-Gleichungen, also für das System $-v \Delta u+\nabla p=f$ zusammen mit $\operatorname{div} u=0$ in $\Omega$ und Randwerten $u=0$ auf $\partial \Omega$ kann man die Bedingung $\operatorname{div} u=0$ als Nebenbedingung in einem Extremalproblem für die kinetische Energie betrachten; der entsprechende Lagrangesche Multiplikator ist dann $\nabla p$. Andererseits gibt es in (1)-(2) keine Zeitableitung für $p$, so dass mit einer Lösung $p(x, t)$ (und $u(x, t)$ ) auch $p(x, t)+q(t)$ für jede beliebige Funktion $q(t)$ ebenfalls eine Lösung ist.

Die weiter unten $\mathrm{zu}$ beschreibenden mathematischen Schwierigkeiten der Navier-Stokes-Gleichungen treten nicht auf, wenn man entweder die Nichtlinearität $u \cdot \nabla u$ oder den Term $\nabla p$ zusammen mit der Divergenzbedingung an $u$ wegläßt. Mit anderen Worten, die Probleme hängen eng mit der Nichtlinearität und der Inkompressibilität zusammen; Gegenbeispiele zur Regularität müssen also alle Terme in den instationären Navier-Stokes-Gleichungen geeignet ausbalanzieren. 
Es gibt nur sehr wenige explizite Lösungen der NavierStokes-Gleichungen, die zudem für spezielle Geometrien konstruiert sind: die Hagen-Poiseuille-Strömung im unendlichen Kanal und die Taylor-Couette-Strömung im rotierenden Zylinderspalt. Beiden Beispielen ist gemeinsam, dass der Term $u \cdot \nabla u$ verschwindet. Die sogenannte Hamel-Strömung im zweidimensionalen Keil mit inflow und outflow ist halb-explizit. Lösungen der linearen StokesGleichung in beschränkten und Außenraumgebieten wurden bereits zu Anfang des 19. Jahrhunderts mit der Methode der Randintegralgleichungen konstruiert. Die instationären Navier-Stokes-Gleichungen im $\mathbb{R}^{3}$ wurden von C. W. Oseen $[31, \S \S 7 \cdot 4-7 \cdot 5]$ mit Hilfe eines Reihenansatzes gelöst. Allerdings liefert dieser Zugang nur ein kleines Existenzintervall $\left[0, T_{0}\right]$, und Oseen bemerkt, dass ein anschließender iterativer Prozess mit einer Folge von Intervallen $\left[T_{0}, T_{1}\right]$, $\left[T_{1}, T_{2}\right], \ldots$ eine Lösung vermutlich nur in einem endlichen Zeitintervall liefern wird. Das Problem in seinen und auch anderen Ansätzen war die Benutzung von klassischen Funktionenräumen z. B. vom Typ $C^{2}$, in denen auch bis heute keine globalen a priori-Abschätzungen gefunden worden sind.

Geeignete globale a priori-Abschätzungen wurden erstmals von J. Leray [28] mit Hilfe der Energie-(Un)gleichung gefunden, die man durch Testen der Navier-Stokes-Gleichung (1) (hier im Fall $f=0$ ) mit der Lösung $u$ findet: multipliziert man (1) skalar mit $u$, führen partielle Integrationen auf die Gleichung

$$
\frac{\mathrm{d}}{\mathrm{d} t} \frac{1}{2} \int_{\Omega}|u(x, t)|^{2} \mathrm{~d} x+v \int_{\Omega}|\nabla u(x, t)|^{2} \mathrm{~d} x=0,
$$

da die Integrale $\int_{\Omega} \nabla p \cdot u \mathrm{~d} x$ und $\int_{\Omega}(u \cdot \nabla u) \cdot u \mathrm{~d} x=\frac{1}{2} \int_{\Omega} u$. $\nabla|u|^{2} \mathrm{~d} x$ wegen der Bedingungen $\left.u\right|_{\partial \Omega}=0$ und $\operatorname{div} u=0$ verschwinden. Eine weitere Integration bzgl. der Zeit auf $[0, t]$ liefert die Energiegleichung

$$
\frac{1}{2}\|u(t)\|_{2}^{2}+v \int_{0}^{t}\|\nabla u\|_{2}^{2} \mathrm{~d} \tau=\frac{1}{2}\|u(0)\|_{2}^{2}
$$

hierbei ist $\|\cdot\|_{2}$ die $L^{2}(\Omega)$-Norm. Wegen $u(0)=u_{0} \in L^{2}(\Omega)$ gilt für die Lösung $u$

$$
u \in L^{\infty}\left(0, T ; L^{2}(\Omega)\right), \quad \nabla u \in L^{2}\left(0, T ; L^{2}(\Omega)\right),
$$

d. h., das Geschwindigkeitsfeld $u$ hat eine für alle Zeiten beschränkte kinetische Energie $\frac{1}{2}\|u(t)\|_{2}^{2}$ sowie eine beschränkte Dissipationsenergie $v \int_{0}^{t}\|\nabla u\|_{2}^{2} \mathrm{~d} \tau$. Die Energiegleichung (3) war das entscheidende neue Argument, das Leray zur Konstruktion globaler schwacher Lösungen führte, der sog. solutions turbulentes.
Ein divergenzfreies Vektorfeld $u$ in der Leray-Hopf-Klasse

$$
\mathcal{L H}_{T}=L^{\infty}\left(0, T ; L_{\sigma}^{2}(\Omega)\right) \cap L^{2}\left(0, T ; H_{0}^{1}(\Omega)\right)
$$

heißt schwache Lösung im Sinne von Leray und Hopf der Navier-Stokes-Gleichungen (1)-(2) mit der Randbedingung $\left.u\right|_{\partial \Omega}=0$ und Anfangswert $u_{0} \in L_{\sigma}^{2}(\Omega)$, falls für alle glatten divergenzfreien Vektorfelder $\varphi$ mit kompaktem Träger in $\Omega \times[0, T)$

$$
\begin{aligned}
-\int_{0}^{T} \int_{\Omega} u \cdot \varphi_{t} \mathrm{~d} x \mathrm{~d} \tau+v \int_{0}^{T} \int_{\Omega} \nabla u: \nabla \varphi \mathrm{d} x \mathrm{~d} \tau \\
\quad-\int_{0}^{T} \int_{\Omega} u \otimes u: \nabla \varphi \mathrm{d} x \mathrm{~d} \tau=\int_{\Omega} u_{0}(x) \cdot \varphi(0, x) \mathrm{d} x
\end{aligned}
$$

gilt. Zudem soll $u$ für alle $t \in[0, T)$ die folgende Energieungleichung erfüllen:

$$
\frac{1}{2}\|u(t)\|_{2}^{2}+v \int_{0}^{t}\|\nabla u\|_{2}^{2} \mathrm{~d} \tau \leq \frac{1}{2}\left\|u_{0}\right\|_{2}^{2}
$$

Hier ist $L_{\sigma}^{2}(\Omega)$ die Menge aller schwach divergenzfreien Vektorfelder $v$ in $L^{2}(\Omega)$ mit verschwindender Normalkomponente $v \cdot n=0$ auf $\partial \Omega$. Ferner besteht $H_{0}^{1}(\Omega)$ aus $L^{2}$ Vektorfeldern $v$ mit $\left.v\right|_{\partial \Omega}=0$ und schwachem Gradienten $\nabla v \in L^{2}(\Omega)$.

Schwache Lösungen der Navier-Stokes-Gleichungen im Sinne von Leray und Hopf existieren für alle beschränkten und sogar für alle unbeschränkten Gebiete. Die klassische Konstruktionsmethode ist das Galerkin-Verfahren, in dem das nichtlineare System (1)-(2) durch eine Folge von nichtlinearen $(N \times N)$-ODE-Systemen erster Ordnung bzgl. $t$ ersetzt wird, die eine Folge von approximativen Lösungen $\left(u_{N}\right)_{N \in \mathbb{N}}$ liefert. Mit der Energiegleichung (3) für $u_{N}$ zeigt man, dass die Folge $\left(u_{N}\right)$ in der Leray-Hopf-Klasse $\mathcal{L} \mathcal{H}_{T}$ beschränkt ist. Deshalb besitzt $\left(u_{N}\right)$ eine schwach bzw. schwach-* konvergente Teilfolge, die wieder mit $\left(u_{N}\right)$ bezeichnet wird, so dass

$$
u_{N} \stackrel{*}{\rightarrow} u \text { in } L^{\infty}\left(0, T ; L_{\sigma}^{2}(\Omega)\right), \quad u_{N} \rightarrow u \text { in } L^{2}\left(0, T ; H_{0}^{1}(\Omega)\right) .
$$

Leider gestattet es die schwache Konvergenz nicht, in der Energiegleichung (3) für $u_{N}$ zur Grenze $N \rightarrow \infty$ zu gehen. Als Beispiel für dieses Phänomen betrachte man die (Folge der) Einheitsvektoren $e_{N}=\left(\delta_{j N}\right)_{j \in \mathbb{N}}$ im Folgenraum $l^{2}(\mathbb{N})$; offensichtlich gilt $e_{N} \rightarrow e=0$ in $l^{2}(\mathbb{N})$ und $\|e\|_{2}<$ $\liminf _{N \rightarrow \infty}\left\|e_{N}\right\|_{2}$. Jedoch garantiert die schwache Unterhalbstetigkeit bzgl. der Norm noch die Energieungleichung (5) für den schwachen Grenzwert $u$. Tatsächlich ist es ein offenes Problem, ob jede schwache Lösung $u$ des Variationsproblems (4) der Energiegleichung (3) oder auch nur der Energieungleichung (5) genügt.

Der entscheidende Schritt beim Grenzübergang $u_{N} \rightarrow u$ betrifft den nichtlinearen Term $u_{N} \cdot \nabla u_{N}$; für diesen Schritt 
ist ein Kompaktheitsargument nötig, welches auf Eigenschaften der Zeitableitungen $\partial_{t} u_{N}$ beruht. Das GalerkinVerfahren wurde z. B. von E. Hopf [22] auf beliebigen Gebieten $\Omega \subset \mathbb{R}^{3}$ benutzt. Dagegen arbeitete Leray im Ganzraumfall $\Omega=\mathbb{R}^{3}$, in dem geeignete a priori-Abschätzungen auf Eigenschaften des Wärmeleitungskerns beruhen.

Grundlegende Eigenschaften schwacher Lösungen im $\mathbb{R}^{2}$ und $\mathbb{R}^{3}$

Mathematisch ist es sinnvoll, das Navier-Stokes-System (1)(2) auch in zwei Dimensionen zu analysieren. Ein Geschwindigkeitsfeld $u=\left(u_{1}, u_{2}, u_{3}\right)$ mit $u_{3} \equiv 0$ und von der dritten Variablen $x_{3}$ unabhängigen Komponenten $u_{1}, u_{2}$ kann zusammen mit $p=p\left(x_{1}, x_{2}, t\right)$ als Lösung von (1)-(2) in einem Gebiet $\Omega \subset \mathbb{R}^{2}$ betrachtet werden.

Im Folgenden vergleichen wir die Eigenschaften schwacher Lösungen im $\mathbb{R}^{d}$ mit $d=2$ und $d=3$, gehen zurück zur angestrebten Energiegleichung (3) und testen a posteriori die Navier-Stokes-Gleichungen nochmals mit der schwachen Lösung $u$. Der kritische Term ist die Nichtlinearität $u \cdot \nabla u$, welche zum Integral

$$
\int_{0}^{T} \int_{\Omega}(u \cdot \nabla u) \cdot u \mathrm{~d} x \mathrm{~d} t
$$

führt. Für (fast) jeden Zeitpunkt $t$ gilt $\int_{\Omega}(u \cdot \nabla u) \cdot u \mathrm{~d} x=0$. Für die Existenz des Integrals (6) muss aber wegen $\nabla u \in$ $L^{2}\left(0, T ; L^{2}(\Omega)\right)$ mehr oder weniger $u \in L^{4}\left(L^{4}\right)$ gelten. Im Fall $d=2$ läßt sich diese Eigenschaft leicht mit Hilfe von Sobolevschen Einbettungssätzen verifizieren. Somit gilt im $\mathbb{R}^{2}$ die Energiegleichung an Stelle der unphysikalischen Energieungleichung. Ebenso kann man Eindeutigkeit und Regularität schwacher Lösungen im $\mathbb{R}^{2}$ zeigen, wenn die Nichtlinearität $u \cdot \nabla u=\operatorname{div}(u \otimes u)$ als Kraftterm mit $u \otimes u \in L^{2}\left(L^{2}\right)$ aufgefasst wird.

Im $\mathbb{R}^{3}$ gilt ebenfalls $u(t) \in L^{4}(\Omega)$ für fast alle $t$; genauer gilt die Gagliardo-Nirenberg-Ungleichung

$$
\|u(t)\|_{4} \leq\|u(t)\|_{2}^{1 / 4}\|\nabla u(t)\|_{2}^{3 / 4} .
$$

Wegen $u \in L^{\infty}\left(L^{2}\right)$ folgt $u \in L^{4}\left(L^{4}\right)$, falls $\nabla u \in L^{3}\left(L^{2}\right)$, welches aber für eine schwache Lösung im Allgemeinen nicht gilt. Die für die Definition der Leray-Hopf-Lösungsklasse $\mathcal{L H}_{T}$ benutzte Energiegleichung kann also a posteriori nicht verifiziert werden. Zudem hat der Term $u \cdot \nabla u$ als Krafterm auf der rechten Seite zu schlechte Eigenschaften, um Eindeutigkeit und Regularität zu garantieren.

Es gibt eine weitere mathematische und physikalische Größe mit verschiedenen Eigenschaften im $\mathbb{R}^{2}$ und $\mathbb{R}^{3}$, nämlich die Wirbelstärke

$$
\omega=\operatorname{curl} u=\left(\partial_{2} u_{3}-\partial_{3} u_{2}, \partial_{3} u_{1}-\partial_{1} u_{3}, \partial_{1} u_{2}-\partial_{2} u_{1}\right) \in \mathbb{R}^{3},
$$

die der Wirbeltransportgleichung

$$
\omega_{t}-v \Delta \omega+u \cdot \nabla \omega-\omega \cdot \nabla u=0
$$

genügt. Für eine ebene Strömung, $d=2$, verschwinden die partielle Ableitung $\partial_{3}$ und die Komponenten $\omega_{1}, \omega_{2}$ von $\omega$, so dass die dritte Komponente $\omega_{3}$ bereits die Wirbelstärke definiert. Die Wirbelstärke als Vektor zeigt im zweidimensionalen Fall stets in die $x_{3}$-Richtung und ist dementsprechend stark eingeschränkt. Zudem verschwindet der Term $\omega \cdot \nabla u=\omega_{3} \partial_{3} u$ in (7). Deshalb ist die skalare Größe $\omega_{3}$ Lösung der Konvektions-Diffusions-Gleichung $\left(\omega_{3}\right)_{t}-v \Delta \omega_{3}+u \cdot \nabla \omega_{3}=0$, für die formal das Maximumund Minimumprinzip gilt. Im Fall $d=3$ ist die Wirbelstärke ein beliebig im Raum orientierter Vektor, und der Term $\omega_{3}$ wird unter vereinfachenden Annahmen durch die Transportgleichung $\partial_{t} \omega_{3}-\omega_{3} \partial_{3} u_{3} \approx 0$ gesteuert. Der vortex stretching term $\omega \cdot \nabla u$ kann aufgrund des dritten Freiheitsgrades im $\mathbb{R}^{3}$ zum Anwachsen eines Wirbels entlang der $x_{3}$-Achse führen. Allerdings ist es nicht die Größe $|\omega|$, die zu Singularitäten führen könnte, sondern nach P. Constantin und Ch. Fefferman [11] der mögliche schnelle Wechsel der Orientierung des Vektors $\omega$ im Raum.

\section{Regularität schwacher Lösungen und Serrin-Zahlen}

Die im vorigen Abschnitt angesprochenen Unterschiede zwischen $d=2$ und $d=3$ können formal leicht mit Hilfe der dimensionsabhängigen Serrin-Zahl

$$
\mathcal{S}(v)=\frac{2}{s}+\frac{d}{q}, \quad v \in L^{s}\left(0, T ; L^{q}(\Omega)\right), \Omega \subset \mathbb{R}^{d},
$$

angegeben werden. Ein intrinsisches Argument zur SerrinBedingung ist die Skaleninvarianz: Für eine Lösung $u, p$ der Navier-Stokes-Gleichungen ist auch

$$
u_{\lambda}(x, t)=\lambda u\left(\lambda x, \lambda^{2} t\right), \quad p_{\lambda}(x, t)=\lambda^{2} p\left(\lambda x, \lambda^{2} t\right)
$$

für jedes $\lambda>0$ eine Lösung von (1)-(2) mit dem gleichen Viskositätskoeffizienten $v$. Diese Eigenschaft garantiert, dass eine „Lösung“ aus einem Windkanalexperiment für ein skaliertes Modell (z. B. Umströmung eines verkleinerten Tragflügelmodells) mit Hilfe von (8) in eine Lösung der realen Welt umgerechnet werden kann. Nun sind die Normen von $u$ und $u_{\lambda}$ in $L^{s}\left(L^{q}\right)$ genau im Fall $\frac{2}{s}+\frac{3}{q}=1$ von $\lambda$ unabhängig. Deshalb wird der Raum $L^{\mathcal{S}}\left(L^{q}\right)$ mit $\mathcal{S}=1$ skaleninvariant genannt. Mit anderen Worten, man kann die Norm einer zu untersuchenden Lösung nicht durch Skalierung „kleinrechnen", um dadurch bessere Ergebnisse zu erzielen. Analysis in solchen Funktionenräumen ist üblicherweise schwieriger, liefert aber in gewissem Sinne optimale Resultate.

Nun hat eine schwache Lösung $u \in L^{\infty}\left(L^{2}\right)$ die SerrinZahl $\mathcal{S}(u)=\frac{d}{2}$. Das gleiche Ergebnis erhält man (formal) mit Hilfe Sobolevscher Einbettungssätze für $\nabla u \in L^{2}\left(L^{2}\right)$. Die zum Beweis der Energiegleichung erforderliche Integrabilität $u \in L^{4}\left(L^{4}\right)$ lautet demnach im $2 d$-Fall $\mathcal{S}(u)=1$, welches für jedes $u \in \mathcal{L H}_{T}$ erfüllt ist, aber $\mathcal{S}(u)=\frac{5}{4}<\frac{3}{2}$ im Fall $d=3$.

Eine typische Forderung an schwache Lösungen ist, dass alle in der Navier-Stokes-Gleichung benutzten Ableitungen auch als schwache Ableitungen existieren, z. B. soll in Verallgemeinerung der Leray-Hopf-Klasse

$$
u \in L^{\infty}\left(0, T ; H^{1}(\Omega)\right) \cap L^{2}\left(0, T ; H^{2}(\Omega)\right)
$$

für reguläre Lösungen gelten, also $\mathcal{S}(u) \leq 1$. Tatsächlich erhält man dieses Ergebnis wie bereits oben erwähnt stets im 
Fall $d=2$, im Fall $d=3$ jedoch auch bei glatten Anfangswerten $u_{0}$ und äußeren Kräften $f$ nur auf kleinen Zeitintervallen $\left(0, T^{\prime}\right) \subset(0, T)$. Eine schwache Lösung ist jedoch regulär, wenn geeignete Zusatzvoraussetzungen erfüllt sind (conditional regularity). Die klassische Bedingung an $u$ ist in jeder Raumdimension die Serrin-Bedingung $\mathcal{S}(u)=1$.

Genauer gelten die folgenden Aussagen: Ist $u \in L^{S}\left(L^{q}\right)$ mit $\mathcal{S}(u)=1$ und $2<s<\infty$, wird die Regularität von $u$ durch die Regularität der Daten und des Randes $\partial \Omega$ bestimmt. So kann $C^{\infty}$-Regularität bis zum Rand gezeigt werden, wenn $\partial \Omega$ von der Klasse $C^{\infty}$ ist; für weitere Resultate verweisen wir auf die Monographie von $\mathrm{H}$. Sohr [34, Theorem V. 1.8.1, V. 1.8.2]. Die wesentliche Idee des Induktionsbeweises ist die Abschätzung der $L^{4}\left(L^{4}\right)$-Norm von $u$ $\left(\mathcal{S}(u)=\frac{5}{4}\right)$ durch die $\|u\|_{L^{s}\left(L^{q}\right)}$-Norm $(\mathcal{S}(u)=1)$ multipliziert mit Normen der Leray-Hopf-Klasse $\left(\mathcal{S}(u)=\frac{3}{2}\right)$ und beruht somit auf der Hölder-Ungleichung in Raum und Zeit.

Analog gilt das folgende weak-strong uniqueness-Resultat, s. Serrin [33]: Sind $u$ und $v$ schwache Lösungen zu den gleichen Daten, gilt $\mathcal{S}(u)=1$ mit $2<s<\infty$ und genügt $v$ der Energie-Ungleichung, stimmen $u$ und $v$ überein.

Der Grenzfall $s=\infty, q=3$ ist wesentlich schwieriger, da der Raum $L^{\infty}(0, T)$ weder reflexiv noch separabel ist. Das entsprechende Regularitätsproblem wurde 2003 durch L. Escauriaza, G. Seregin, und V. Šverák [13] im Fall $\Omega=\mathbb{R}^{3}$ gelöst; die neue Idee ist die backward uniqueness property der Wärmeleitungsgleichung bzw. -ungleichung angewandt auf die Wirbelstärke.

Neben der Serrin-Bedingung $\mathcal{S}(u)=1$ gibt es eine Vielzahl von Ergebnissen zur conditional regularity, also Bedingungen an $u, \nabla u$, oder an spezielle Komponenten von $u, \nabla u$, von $\omega$, an den Druck $p$ und weitere physikalische Größen. Diese Ergebnisse sind eher von theoretischem Interesse, da in der Praxis nicht überprüft werden kann, ob eine schwache Lösung die geforderte Zusatzbedingung erfüllt. Die bisher unüberwindbare Lücke zwischen den Serrin-Zahlen $\mathcal{S}(u)=\frac{3}{2}$ für schwache Lösungen im $\mathbb{R}^{3}$ und $\mathcal{S}(u)=1$ für reguläre Lösungen bleibt bestehen.

\section{Konstruktion regulärer Lösungen}

Obwohl es viele klassische Methoden zur Konstruktion globaler schwacher Lösungen gibt, sind diese Lösungen im Allgemeinen nur auf einem kleinen Zeitintervall regulär. Eine andere Idee ist die direkte Konstruktion regulärer Lösungen. Beim Galerkinverfahren nimmt man z. B. eine Orthonormalbasis aus Eigenfunktionen des Stokes-Operators $A=-P \Delta$ in einem beschränkten Gebiet. Dabei ist $P: L^{2}(\Omega)^{d} \rightarrow L_{\sigma}^{2}(\Omega)$ die Helmholtz-Projektion, die ein Vektorfeld auf seinen divergenzfreien Anteil projiziert, so dass $u-P u$ ein Gradientenfeld ist. Die mathematische Arbeit besteht darin, geeignete $a$ priori-Abschätzungen von $\nabla u$ und $A u$ zu finden, wenn (1) z. B. mit $A u$ statt $u$ getestet wird. Der kritische Term $\int_{\Omega}(u \cdot \nabla u) \cdot A u \mathrm{~d} x$ führt dazu, dass die Dissipationsrate $y(t):=\|\nabla u(t)\|_{2}^{2}$ die Differentialungleichung $y^{\prime} \leq C v^{-3} y^{3}$ erfüllt. Somit ist $y(t)$ mindestens auf dem Intervall $\left(0, T_{0}\right)$ mit $T_{0}=c v^{3}\left\|\nabla u_{0}\right\|_{2}^{-4}$ endlich; man beachte die Abhängigkeit von $T_{0}$ vom Anfangswert und der Viskosität.
J. Leray [28] hat bereits für eine für $t \rightarrow T_{0}-$ singulär werdende Lösung $\left(\mathrm{im} \mathbb{R}^{3}\right)$ gezeigt, dass sie mit der Rate

$$
\|\nabla u(t)\|_{2} \geq \frac{c v^{3 / 4}}{\left(T_{0}-t\right)^{1 / 4}}, \quad t \rightarrow T_{0^{-}}
$$

divergiert. Außerdem gibt es wegen der Dissipation (Reibung) für jede globale schwache Lösung $u$ ein $T_{\infty} \geq T_{0}$, so dass $u$ auf $\left(T_{\infty}, \infty\right)$ regulär ist. Diese beiden Fakten sind die Bausteine des Lerayschen Struktursatzes: Für jede globale schwache Lösung gibt es endlich oder abzählbar viele Regularitätsintervalle $I_{j} \subset\left(0, T_{\infty}\right)$, so dass $u$ auf jedem Interval $I_{j}$ und auf $\left(T_{\infty}, \infty\right)$ regulär ist; außerdem ist das Komplement $[0, \infty) \backslash \bigcup_{j} I_{j} \backslash\left(T_{\infty}, \infty\right)$ eine Lebesgue-Nullmenge, und es gilt sogar

$$
\mathcal{H}^{1 / 2}\left([0, \infty) \backslash \bigcup_{j} I_{j} \backslash\left(T_{\infty}, \infty\right)\right)=0
$$

für das $\frac{1}{2}$-dimensionale Hausdorffmaß $\mathcal{H}^{1 / 2}$, siehe z. B. [19]. Dennoch könnte diese Menge überabzählbar viele Zeitpunkte enthalten, auch wenn noch kein Beispiel bekannt ist, in dem überhaupt ein einziger solcher Zeitpunkt existiert. Ähnliche Ergebnisse gelten für schwache Lösungen auf beschränkten und Außenraumgebieten, falls eine sog. starke Energieungleichung erfüllt ist.

\section{Halbgruppenmethoden}

Eine weitere Methode zur Konstruktion regulärer Lösungen basiert auf analytischer Halbgruppentheorie. Bekanntlich erzeugt der Stokes-Operator $A=-P \Delta$ sowohl für beschränkte und Außenraumgebiete als auch für Schichten (layer), Röhren und Halbräume (sowie deren Varianten) eine beschränkte analytische Halbgruppe $e^{-t A}, t \geq 0$. Dann führt die Duhamel-Formel auf die nichtlineare Integralgleichung

$$
u(t)=e^{-v t A} u_{0}-\int_{0}^{t} e^{-v(t-\tau) A} P(u \cdot \nabla u)(\tau) \mathrm{d} \tau .
$$

Für die Analysis dieser Gleichung benötigt man die Eigenschaft der maximalen Regularität des Stokes-Operators, d. h. die a priori-Abschätzung

$$
\left\|u_{t}\right\|_{L^{s}\left(0, T, L^{q}\right)}+\|A u\|_{L^{s}\left(0, T ; L^{q}\right)} \leq c\|f\|_{L^{s}\left(0, T ; L^{q}\right)}
$$

für Lösungen des instationären Stokes-Systems

$$
u_{t}+v A u=f \quad \text { in } \Omega \times(0, T), \quad u(0)=0 .
$$

Unter geeigneten Annahmen an $\Omega$ gilt die Abschätzung (11) für alle $1<s, q<\infty$. Erste Ergebnisse diesen Typs stammen von V.A. Solonnikov [35] für $s=q$; für einen modernen, funktionalanalytischen Beweis s. M. Geissert et al. [20]. Mit Hilfe der maximalen Regularität und gebrochener Potenzen des Stokes-Operators liefert der Banachsche Fixpunktsatz schließlich eine Lösung $u$ von (10). Um jedoch eine kontrahierende Selbstabbildung zu erhalten, müssen Kleinheitsannahmen an den Anfangswert oder die Länge des Existenzintervals $[0, T)$ gestellt werden.

Diese Methode beruht entscheidend auf der $L^{q}$-Theorie, $q \neq 2$, der Helmholtz-Projektion und des Stokes-Operators. Es gibt jedoch ein berühmtes Gegenbeispiel von M. E. Bogovskiǐ (1986) eines glatten unbeschränkten Gebietes, auf 
dem die Helmholtz-Projektion die üblichen Abbildungseigenschaften nicht besitzt, so dass der Stokes-Operator $A=-P \Delta$ nicht wohldefiniert ist. Andererseits gestatten Hilbertraummethoden für jedes beschränkte und unbeschränkte Gebiet die Definition der Operatoren $P, A$ im $L^{2}$-Raum, s. [34]. Die Kombination einer lokalen $L^{q}$-Theorie und der global geltenden $L^{2}$-Theorie ist der Schlüssel zur Analysis von $P$ und $A$ in allgemeinen glatten unbeschränkten Gebieten in Räumen vom Typ

$$
\tilde{L}^{q}(\Omega)= \begin{cases}L^{2}(\Omega)+L^{q}(\Omega), & 1<q<2 \\ L^{2}(\Omega) \cap L^{q}(\Omega), & 2 \leq q<\infty\end{cases}
$$

s. H. Kozono, H. Sohr und der Autor ([14, 15]).

Selbstverständlich muss für starke Lösungen $u \in$ $L^{s}\left(0, T ; L^{q}(\Omega)\right), \frac{2}{s}+\frac{3}{q}=1$, der Anfangswert $u_{0}$ höhere Regularität als nur $L_{\sigma}^{2}(\Omega)$ haben. Für das Stokes-System (12) mit $f=0$, aber $u(0)=u_{0} \neq 0$, muss die Lösung $u(t)=e^{-t A} u_{0}$ die Bedingung

$$
\int_{0}^{\infty}\left\|e^{-t A} u_{0}\right\|_{q}^{s} \mathrm{~d} t<\infty
$$

erfüllen. Äquivalent dazu sind mit Hilfe der reellen Interpolationstheorie die Forderungen

$$
A^{-1} u_{0} \in\left(L_{\sigma}^{q}(\Omega), \mathcal{D}\left(A_{q}\right)\right)_{1-1 / s, s}
$$

oder - in der Sprache der Besovräume $-u_{0} \in B_{q, s}^{-1+3 / q}(\Omega)$. Dabei ist $L_{\sigma}^{q}(\Omega)=\left\{u \in L^{q}(\Omega): \operatorname{div} u=0\right.$ in $\Omega, u \cdot n=0$ auf $\left.\partial \Omega\right\}$ und $\mathcal{D}\left(A_{q}\right)$ der Definitionsbereich des Stokes-Operators $A$ auf $L_{\sigma}^{q}(\Omega)$. In [16] haben H. Sohr und der Autor gezeigt, dass der optimale (größte) Raum von Anfangswerten, zu denen die nichtlinearen Navier-Stokes-Gleichungen zumindest lokale, starke Lösungen in der Serrin-Klasse besitzen, der „Besovraum“ $B_{q, s}^{-1+3 / q}(\Omega) \cap L_{\sigma}^{2}(\Omega)$ ist. Außerdem läßt sich mit (13) ein $T>0$ angeben, so dass Regularität auf dem Intervall $[0, T)$ vorliegt, s. [17].

\section{Analysis in Grenzfällen skaleninvarianter Räume}

Der klassische Grenzfall von starken Lösungen in $\left.C^{0}[0, T] ; L^{3}(\Omega)\right) \subset L^{\infty}\left(0, T ; L^{3}(\Omega)\right)$ kann weder durch die Galerkin-Methode noch den Halbgrupppensansatz in (10) direkt behandelt werden; ein elementarer Hinweis ist die Tatsache, dass $\|v\|_{L^{\infty}(0, T)}$ nicht notwendigerweise für $T \rightarrow 0$ gegen 0 konvergiert. Dieses Problem wurde erstmalig von $\mathrm{T}$. Kato [24] durch ein zweistufiges Iterationsschema gelöst: Im ersten Schritt wird eine Iteration für $\left(u_{j}\right)_{j \in \mathbb{N}}$ in (10) durchgeführt, so dass

$$
\begin{gathered}
t^{\frac{1}{2}\left(1-\frac{3}{q}\right)} u_{j}(t) \in C\left([0, T] ; L^{q}(\Omega)\right), \\
\left.t^{\frac{1}{2}} \nabla u_{j}(t) \in C[0, T] ; L^{3}(\Omega)\right)
\end{gathered}
$$

gilt. Hier ist $q>3$ beliebig. Nach dem Konvergenzbeweis dieses Schemas wird in einem zweiten Schritt die Konvergenz von $\left(u_{j}\right)$ in $C\left(\left[0, T ; L^{3}(\Omega)\right)\right.$ bewiesen. Der Beweis von
Kato wurde für den Ganzraumfall $\mathbb{R}^{3}$ entwickelt, kann aber für andere Gebiete angepasst werden. Außerdem liefert er für genügend kleine Anfangswerte $u_{0} \in L^{3}(\Omega)$ sogar die Existenz globaler Lösungen.

Der bisher bekannte größte skaleninvariante Raum von Anfangswerten, in dem lokale und/oder globale Lösungen existieren, wurde von $\mathrm{H}$. Koch und D. Tataru [26] gefunden. Für $u_{0} \in B M O^{-1}\left(\mathbb{R}^{3}\right)$, d. h. $u_{0}=\operatorname{div} f$ mit $f \in$ $B M O\left(\mathbb{R}^{3}\right)$, mit kleiner Norm existiert eine globale Lösung $u \in L_{\mathrm{loc}}^{\infty}\left((0, \infty) ; \mathbb{R}^{3}\right)$ mit skaleninvarianter Norm

$$
\sup _{t>0} t^{\frac{1}{2}}\|u(t)\|_{L^{\infty}}+\left(\sup _{x \in \mathbb{R}^{3}, R>0} \frac{1}{R^{3}} \int_{0}^{R^{2}} \int_{\{y:|y-x|<R\}}|u|^{2} \mathrm{~d} y \mathrm{~d} \tau\right)^{1 / 2} .
$$

Der größtmögliche skaleninvariante Raum für die NavierStokes-Gleichungen ist der homogene Besovraum $\dot{B}_{\infty, \infty}^{-1}$ ([6, Proposition 7]), der den Raum $B M O^{-1}$ enthält; deshalb sind in diesem Raum formulierte Bedingungen an Lösungen die interessantesten. Die Navier-Stokes-Gleichungen sind im Raum $\dot{B}_{\infty, \infty}^{-1}$ jedoch nicht wohlgestellt, s. J. Bourgain und N. Pavlović [1]: Für jedes $\delta>0$ existieren ein Anfangswert $u_{0}$ in der Schwartz-Klasse $\mathcal{S}\left(\mathbb{R}^{3}\right)$, ein $T_{\delta} \in(0, \delta)$ und dazu eine Lösung $u$ mit

$$
\left\|u_{0}\right\|_{\dot{B}_{\infty, \infty}^{-1}} \leq \delta \quad \text { and }\left\|u\left(T_{\delta}\right)\right\|_{\dot{B}_{\infty, \infty}^{-1}} \geq \frac{1}{\delta}
$$

Dieses Ergebnis wurde von T. Yoneda [40] und B. Wang [38] auf die Räume $\dot{B}_{\infty, r}^{-1}$ mit $1 \leq r<\infty$ verallgemeinert. Da $B_{\infty, \infty}^{-1}$ größer als $\dot{B}_{\infty, \infty}^{-1}$ ist, werden Anfangswerte in $B_{\infty, \infty}^{-1}$ nicht betrachtet, wohl aber Regularitätsfragen. Z.B. bewiesen A. Cheskidov und R. Shvydkoy ([10]), dass eine schwache Leray-Hopf-Lösung $u \in C^{0}\left((0, T] ; B_{\infty, \infty}^{-1}\right)$ oder mit genügend kleinen Sprüngen in $\operatorname{der} B_{\infty, \infty}^{-1}$-Norm regulär ist. Andererseits können schwache Lösungen vom LerayHopf-Typ in $t=0$ bzgl. der Norm von $B_{\infty, \infty}^{-1}$ unstetig sein: Cheskidov und Shvydkoy [9] konstruieren Anfangswerte $u_{0} \in \bigcap_{1<q \leq \infty} B_{q, \infty}^{3 / q-1} \cap L_{\sigma}^{2}$, so dass für jede schwache Lösung $u$ mit $u(0)=u_{0}$

$$
\limsup _{t \rightarrow 0+}\left\|u(t)-u_{0}\right\|_{B_{\infty, \infty}^{-1}} \geq \delta
$$

gilt. Dieses Ergebnis ist das Gegenstück zu den positiven Resultaten in $[16,17]$.

Dennoch gibt es Anfangswerte mit beliebig großen Normen in $\dot{B}_{\infty, \infty}^{-1}$, die globale glatte Lösungen erzeugen. Diese speziell konstruierten Anfangswerte sind z. B. in einer Variablen mit Periode $\frac{1}{\varepsilon}$ stark oszillierend, s. Arbeiten von J.-Y. Chemin, I. Gallagher und M. Paicu [7], [8].

\section{Lokale Regularität und Singularitäten}

Im Gegensatz zu den bisherigen Resultaten zur Regularität global im Raum wie im Lerayschen Struktursatz, s. (9), untersucht die lokale Regularitätstheorie Punkte $z_{0}=\left(x_{0}, t_{0}\right) \in$ $\mathbb{R}^{4}$, so dass $u$ für keine Umgebung $U \subset \mathbb{R}^{4}$ von $z_{0}$ in $L^{\infty}(U)$ liegt. Aufgrund des lokalen Charakters muss die schwache Lösung eine lokalisierte Version der Energieungleichung er- 
füllen und wird geeignete schwache Lösung genannt. Der Existenzbeweis solcher Lösungen verlangt eine sorgfältige Konstruktion, da aufgrund der Benutzung von Abschneidefunktionen der Druckterm abgeschätzt werden muss. Geeignete schwache Lösungen existieren sowohl für beschränkte und Außenraumgebiete als auch für allgemeine unbeschränkte glatte Gebiete, s. [5, 14, 15].

Das berühmte Ergebnis von L. Caffarelli, R. Kohn und L. Nirenberg [5] besagt, dass die Menge der singulären Raumzeitpunkte $\mathcal{S} \subset \mathbb{R}^{4}$ einer geeigneten schwachen Lösung $u$ die Hausdorff-Bedingungen $\operatorname{dim}_{\mathcal{H}}(\mathcal{S})=1$ und sogar

$$
\mathcal{H}^{1}(\mathcal{S})=0
$$

erfüllt. Deshalb enthält $\mathcal{S}$ keine Geradenstücke und keine glatten Wege. Folglich entwickeln axialsymmetrische Strömungen Singularitäten nur auf der Rotationsachse.

Die Aussage (14) ist eine integrierte Version der lokalen Regularitätsanalyse im Raum-Zeit-Zylinder $Q_{r}\left(z_{0}\right)=$ $B_{r}\left(x_{0}\right) \times\left(t_{0}, t_{0}-r^{2}\right)$ und benutzt entweder das Kriterium

$$
\limsup _{r \rightarrow 0+} \frac{1}{r} \iint_{Q_{r}\left(z_{0}\right)}|\nabla u|^{2} \mathrm{~d} x \mathrm{~d} \tau<\varepsilon_{0}
$$

oder

$$
\frac{1}{r^{2}} \iint_{Q_{r}\left(z_{0}\right)}|u|^{3} \mathrm{~d} x \mathrm{~d} \tau<\varepsilon_{0} .
$$

Dabei ist der Parameter $\varepsilon_{0}>0$ eine absolute Konstante und die obigen Integrale sind invariant bzgl. der Skalierung (8). Außerdem kann der Term $\nabla u$ durch die Wirbelstärke $\omega=\operatorname{rot} u$ ersetzt werden. Zu Beweisen und weiteren Ergebnissen verweisen wir auf $[5,27,29,39]$.

Eine klassische Idee von Leray [28] zur Konstruktion schwacher Lösungen mit Singularitäten beruht auf dem Ansatz selbstähnlicher Lösungen der Gestalt

$$
u(x, t)=\frac{1}{\sqrt{-t}} V\left(\frac{x}{\sqrt{-t}}\right)
$$

mit $V \in L^{3}\left(\mathbb{R}^{3}\right)$; derartige Lösungen werden für $t \rightarrow 0-$ singulär. Da $u$ eine schwache Lösung ist, genügt das Vektorfeld $V(y)$ einer $t$-unabhängigen modifizierten Navier-StokesGleichung mit einem $y$-abhängigen Zusatzterm. Die Aufgabe, ein solches $V \neq 0$ und damit eine singuläre schwache Lösung zu finden, blieb in [28] offen und wurde erst 1996 von J. Nečas, M. Růžička und V. Šverák ([30]) negativ beantwortet. Für den Fall $V \in L^{q}\left(\mathbb{R}^{3}\right), q>3$, verweisen wir auf [37].

Im punktweisen Sinne sind die bisher betrachteten Singularitäten mehr oder weniger von der Form $|u(x, t)| \sim$ $\frac{c}{|x|+\sqrt{-t}}$ (Typ I-Singularitäten). Sie können für selbstähnliche und für axialsymmetrische Strömungen ausgeschlossen werden, s. [25]. Alle anderen Singularitäten heißen vom Typ II.

\section{2 \\ Das Gegenbeipiel von Terence Tao}

Eine Lösung mit Singularität in endlicher Zeit bei glattem Anfangswert wurde von T. Tao [36] für ein modifiziertes gemitteltes Navier-Stokes-System im $\mathbb{R}^{3}$ konstruiert. Dieses
Gegenbeispiel basiert auf einer blow-up-Lösung der EulerGleichungen, wobei der glättende Dissipationsterm $-\Delta u$ nun als Störterm agiert, aber schließlich dennoch eine Singularität vom Typ II zuläßt. Ausgangspunkt ist ein diskretes dynamisches System skalarer Funktionen $\left(X_{n}(t)\right)_{n \in \mathbb{Z}}$ in der Form

$$
\partial_{t} X_{n}(t)=-\lambda^{2 n \alpha} X_{n}+\lambda^{n-1} X_{n-1}^{2}-\lambda^{n} X_{n} X_{n+1}
$$

mit Parameter $\lambda=1+\varepsilon \approx 1$. In der Endversion ist $X_{n}$ jedoch ein Vektorfeld mit vier Komponenten mit paarweise disjunkten Trägern im Fourierraum, für die Energie immer schneller hin- und hergepumpt wird. Aus den $X_{n}$ wird eine Lösung $u$ über die Reihe $\sum_{n} X_{n}(t) \lambda^{3 n / 5} \psi\left(\lambda^{2 n / 5} x\right)$ mit einer geeigneten Schwartz-Funktion $\psi$ konstruiert. Zur Kontrolle des nichtlinearen Energietransports in (16) muss die Interaktion einzelner Paare $\left(X_{n}, X_{n+1}\right)$ genau gesteuert werden. $\mathrm{Zu}$ diesem $\mathrm{Ziel}$ wird eine komplizierte Modifizierung des bilinearen Operators $B(u, v):=\frac{1}{2}(u \cdot \nabla v+v \cdot \nabla u)$ oder, genauer, des trilinearen Terms

$$
\langle B(u, v), w\rangle_{\mathbb{R}^{3}}=\int_{\mathbb{R}^{3}} \frac{1}{2}(u \cdot \nabla v+v \cdot \nabla u) w \mathrm{~d} x,
$$

vorgenommen. Dazu benutzt Tao Rotationsoperatoren $\mathcal{R}_{\omega} u(x):=R_{\xi} u\left(R_{\xi}^{-1} x\right)$ mit Rotationsachsen $\xi \in \mathbb{R}^{3}$ sowie Multiplier-Operatoren $\mathcal{M} u=\mathcal{F}^{-1} m(\cdot) \mathcal{F} u$. Damit wird der trilinear Term $\langle B(u, v), w\rangle_{\mathbb{R}^{3}}$ durch eine aufwändig gemittelte Trilinearform $\langle\tilde{B}(u, v), w\rangle_{\mathbb{R}^{3}}$ ersetzt, indem Operatoren $\mathcal{M}_{\omega}^{(1)} \mathcal{R}_{\omega}^{(1)}$ auf $u$ angewandt werden; analog verfährt man mit $v$ und $w$. Insgesamt durchläuft die Zufallsvariable $\omega$ eine Teilmenge von $S O\left(\mathbb{R}^{3}\right) \times S O\left(\mathbb{R}^{3}\right) \times S O\left(\mathbb{R}^{3}\right) \times \mathbb{R}^{3} \times \mathbb{R}^{3} \times \mathbb{R}^{3}$. Die entscheidenden Eigenschaften von $\tilde{B}$ sind die Auslöschungseigenschaft $\langle\tilde{B}(u, u), u\rangle_{\mathbb{R}^{3}}=0$ und die für $B$ gültigen Energieabschätzungen. Tao's Beispiel impliziert, dass eine positive Antwort zum Regularitätsproblem entweder auf komplizierteren Abschätzungen und verborgenen Eigenschaften von $B$ beruht, die nicht für $\tilde{B}$ gelten, oder auf einer weiteren Balance zwischen den Termen $u_{t}+u \cdot \nabla u,-\Delta u$ und dem Druck $\nabla p$.

\section{Neue Wege zur Nichteindeutigkeit}

In [23] beschreiben H. Jia und V. Šverák eine Situation, in der Anfangswerte mehrere Lösungen zulassen. Ausgangspunkt ist ein selbstähnlicher Anfangswert $u_{0}$, so dass zu $\sigma u_{0}, 0 \leq \sigma \ll 1$, eine eindeutige globale selbstähnliche Lösung $u_{\sigma}(x, t)=t^{-1 / 2} U_{\sigma}\left(x t^{-1 / 2}\right)$ existiert, vgl. (15). Der Operator $\mathcal{L}_{\sigma}$ für die Anwendung von Verzweigungstheorie ist die von $U_{\sigma}$ erfüllte modifizierte (Navier-)Stokes-Gleichung von Leray. Für $\mathcal{L}_{\sigma}$ müssen geeignete Annahmen an die Eigenwerte in Abhängigkeit von $\sigma$ erfüllt sein, so dass für ein $\sigma_{0}>0$ eine pitchfork-Verzweigung auftritt. Diese theoretischen Annahmen konnten bisher nicht rigoros verifiziert werden. Numerische Tests von J. Guillod und V. Šverák [21] deuten jedoch darauf hin, dass das oben geforderte Eigenwertszenario in speziellen Situationen vorliegt.

Mit der Methode der konvexen Integration haben C. De Lellis und L. Székelyhidi Jr. die Existenz schwacher nichttrivialer Lösungen der ${ }_{3} \mathrm{D}$-Euler-Gleichung in der Klasse 
$C^{\beta}, 0<\beta<\frac{1}{3}$, in Raum und Zeit mit vorgeschriebener kinetischer Energie $E_{\text {kin }}(t) \geq 0$ bewiesen, s. [4, 12]. Diese Ergebnisse wurden in den letzten Jahren auf die NavierStokes-Gleichungen verallgemeinert, wobei der glättende Effekt durch die Dissipation (Reibung) das entscheidende Problem war: es gibt schwache Lösungen im Sinne $u \in C^{0}\left([0, T] ; L^{2}(\Omega)\right)$, so dass $u$ im Allgemeinen weder der Energie(un-)gleichung genügt noch endliche Dissipationsenergie $\int_{0}^{t}\|\nabla u(t)\|_{2}^{2} \mathrm{~d} \tau$ hat. In [2] schreiben T. Buckmaster und V. Vicol eine glatte Funktion $E_{\text {kin }}(t) \geq 0$ vor und konstruieren eine schwache Lösung $u \in C^{0}\left([0, T] ; L^{2}\left(\mathbb{T}^{3}\right) \cap\right.$ $\left.W^{1,1+\beta}\left(\mathbb{T}^{3}\right)\right)$ mit $\beta>2^{-16}$ und $\frac{1}{2}\|u(t)\|_{2}^{2}=E_{\text {kin }}(t)$. Da $E_{\text {kin }}$ nicht als monoton fallend angenommen wurde, ist die schwache Lösung im Allgemeinen nicht physikalisch sinnvoll (physically reasonable). Dieses Ergebnis wurde von den Autoren und M. Colombo in [3] wie folgt verallgemeinert: zu glatten Lösungen $u^{(1)}, u^{(2)}$ der Navier-Stokes-Gleichungen auf $\mathbb{T}^{3} \times[0, T]$ mit $f \equiv 0$ gibt es eine schwache Lösung im obigen Sinne mit $u(0)=u^{(1)}(0)$, die

$$
u \equiv u^{(1)} \text { auf }[0, T / 3] \quad \text { und } u \equiv u^{(2)} \text { auf }[2 T / 3, T]
$$

erfüllt. Außerdem ist die Menge $\mathcal{S}$ der singulären Zeitpunkte klein im Sinne $\mathcal{H}^{1-\beta}(\mathcal{S})=0$. Wählt man insbesondere $u^{(1)} \equiv 0$ und $u^{(2)} \neq 0$, erhält man eine Lösung, die für einige Zeit im Ruhezustand verharrt, bevor sie zu einer nichtverschwindenden glatten Lösung verzweigt. Andererseits kann $u$ mit $u \equiv u^{(1)} \neq 0$ auf $[0, T / 3]$ beginnen, aber ab $t=2 T / 3 \mathrm{im}$ Ruhezustand sein.

Da diese Lösungen nicht die Energieungleichung auf $[T / 3,2 T / 3]$ erfüllen werden, kommt die Frage nach schwachen Lösungen im Sinne von Leray-Hopf mit einem physikalisch sinnvollen Verhalten der kinetischen Energie auf. In einer Teilantwort gibt W. S. Ożański [32] eine stetige, monoton fallende Energiefunktion $E_{\text {kin }}(t)$ vor und konstruiert eine geeignete schwache Lösung $u$, die die lokalisierte Energieungleichung erfüllt und für die zusätzlich $\frac{1}{2}\|u(t)\|_{2}^{2}-E_{\text {kin }}(t)$ beliebig klein ist. Allerdings genügt $u$ nur einer NavierStokes-Ungleichung, d. h., $u$ ist eine Lösung in der LerayHopf-Klasse mit der Eigenschaft, dass das punktweise Skalarprodukt im $\mathbb{R}^{3}$ der Kraft $f$ mit dem Geschwindigkeitsfeld $u$ nicht positiv ist:

$$
f:=u_{t}-v \Delta u+u \cdot \nabla u+\nabla p, \quad f \cdot u \leq 0 .
$$

Damit ist $f$ keine vorgegebene Funktion, sondern erfüllt $a$ posteriori die Ungleichung $f \cdot u \leq 0$; mit anderen Worten: die Kraft $f$ agiert stets gegen die Strömungsrichtung.

\section{Zusammenfassung}

Selbst nach mehr als 85 Jahren, vor denen Leray schwache Lösungen konstruierte, ist das Regularitätsproblem für globale schwache Lösungen der Navier-Stokes-Gleichungen im $\mathbb{R}^{3}$ oder die direkte Konstruktion globaler regulärer Lösungen noch offen; dieses gilt auch nach langer intensiver Arbeit in klassischen $L^{2}$ - und $L^{p}$-Räumen, in Lorentzund Sobolevräumen, mit und ohne Gewichten, moderner Analysis in Besovräumen, sowie Räumen vom Typ BMO, Hardy $\mathcal{H}^{1}$, Triebel-Lizorkin, Morrey, und vielen anderen. Dabei konnte die Lücke zwischen schwachen Lösungen mit
Serrin-Zahl $\mathcal{S}=\frac{3}{2}$ und starken Lösungen mit $\mathcal{S}=1$ nicht geschlossen werden. Andere Ideen zur Konstruktion von Lösungen mit blow-up in endlicher Zeit scheiterten wie z. B. der Ansatz selbstähnlicher Lösungen von Leray oder Lösungen modifizierter Navier-Stokes-Gleichungen (Tao), wobei nicht klar ist, ob und wie die Konstruktion auf das ursprüngliche PDE-System übertragen werden kann. Eine neue Richtung erschließt sich durch Beweise zur Nichteindeutigkeit in einer neuen Klasse schwacher Lösungen. Diese Lösungen mit ihrem unerwarteten Verhalten haben jedoch nicht die üblichen in der Physik geforderten Eigenschaften (Energie-(un-)gleichung, endliche Dissipationsenergie).

\section{Literatur}

[1] J. Bourgain und N. Pavlović, Ill-posedness of the Navier-Stokes equations in a critical space in 3D. J. Funct. Anal. 255 (2008), 2233-2247.

[2] T. Buckmaster und V. Vicol, Nonuniqueness of weak solutions to the Navier-Stokes equation. Ann. of Math. 189 (2019), 101-144.

[3] T. Buckmaster, M. Colombo und V. Vicol, Wild solutions of the Navier-Stokes equations whose singular sets in time have Hausdorff dimension strictly less than 1. arXiv:1809.0060o (2018).

[4] T. Buckmaster, C. de Lellis, L. Székelyhidi Jr. und V. Vicol, Onsager's conjecture for admissible weak solutions. Comm. Pure Appl. Math. 72 (2019), 229-274.

[5] L. Caffarelli, R. Kohn und L. Nirenberg, Partial regularity of suitable weak solutions of the Navier-Stokes equations. Comm. Pure Appl. Math. 35, 771-831 (1982).

[6] M. Cannone, Harmonic analysis tools for solving the incompressible Navier-Stokes equations. In: Handbook of Mathematical Fluid Dynamics, Vol. III, North-Holland, Amsterdam 2004, 161-244.

[7] J.-Y. Chemin und I. Gallagher, Wellposedness und stability results for the Navier-Stokes equations in $\mathbb{R}^{3}$. Ann. Inst. H. Poincaré, Anal. Non Linéaire 26 (2009), 599-624.

[8] J.-Y. Chemin, I. Gallagher und M. Paicu, Global regularity for some classes of large solutions to the Navier-Stokes equations. Ann. of Math. 173 (2011) , 983-1012.

[9] A. Cheskidov und R. Shvydkoy, Ill-posedness of the basic equations of fluid mechanics in Besov spaces. Proc. Amer. Math. Soc. 138 (2010), 1059-1067.

[10] A. Cheskidov und R. Shvydkoy, The regularity of weak solutions of the 3 D Navier-Stokes equations in $B_{\infty, \infty}^{-1}$. Arch. Ration. Mech. Anal. 195 (2010), 159-169.

[11] P. Constantin und Ch. Fefferman, Direction of vorticity und the problem of global regularity for the Navier-Stokes equations. Indiana Univ. Math. J. 42 (1993), 775-789.

[12] C. De Lellis und L. Székelyhidi Jr., Dissipative continuous Euler flows. Invent. Math. 193 (2013), 377-407.

[13] L. Escauriaza, G. Seregin und V. Šverák, $L_{3, \infty}$-solutions of the Navier-Stokes equations und backward uniqueness. Uspekhi Mat. Nauk 58 (2003), 3-44 (Russian), Russian Math. Surveys 58 (2003), 211-250.

[14] R. Farwig, H. Kozono und H. Sohr, An $L^{q}$-approach to Stokes und Navier-Stokes equations in general domains. Acta Math 195 (2005), 21-53.

[15] R. Farwig, H. Kozono und H. Sohr, Stokes semigroups, strong, weak, and very weak solutions for general domains. In: Y. Giga and A. Novotný (eds.), Handbook of Mathematical Analysis in Mechanics of Viscous Fluids. Springer Cham 2018

[16] R. Farwig und H. Sohr, Optimal initial value conditions for the existence of local strong solutions of the Navier-Stokes equations. Math. Ann. 345 (2009), 631-642. 
[17] R. Farwig, H. Sohr und W. Varnhorn, On optimal initial value conditions for local strong solutions of the Navier-Stokes equations, Ann. Univ. Ferrara Sez. VII Sci. Mat. 55 (2009), 89-110.

[18] Ch. Fefferman, Existence and smoothness of the NavierStokes equation. http://www.claymath.org/sites/default/files/ navierstokes.pdf

[19] G.P. Galdi, An introduction to the Navier-Stokes initial-boundary value problem. Fundamental Directions in Mathematical Fluid Mechanics, 1-70, Adv. Math. Fluid Mech., Birkhäuser, Basel, 2000.

[20] M. Geissert, M. Hess, M. Hieber, C. Schwarz und K. Stavrakidis, Maximal $L^{p}-L^{q}$-estimates for the Stokes equation: a short proof of Solonnikov's theorem. J. Math. Fluid Mech. 12 (2010), 47-6o.

[21] J. Guillod, V. Šverák, Numerical investigations of non-uniqueness for the Navier-Stokes initial value problem in borderline spaces. arXiv:1704.00560v1 (2017)

[22] E. Hopf, Über die Anfangswertaufgabe für die hydrodynamischen Grundgleichungen. Math. Nachr. 4 (1951), 213-231.

[23] H. Jia, V. Šverák, Are the incompressible 3 d Navier-Stokes equations locally ill-posed in the natural energy space? J. Funct. Anal. 268 (2015), 3734-3766.

[24] T. Kato, Strong $L^{p}$-solutions of the Navier-Stokes equations in $\mathbb{R}^{m}$, with applications to weak solutions. Math. Z. 187 (1984), $471-480$.

[25] G. Koch, N. Nadirashvili, G. Seregin und V. Šverák, Liouville theorems for the Navier-Stokes equations and applications. Acta Math. 203 (2009), 83-105.

[26] H. Koch und D. Tataru, Well-posedness for the Navier-Stokes equations. Adv. Math. 157 (2001), 22-35.

[27] O. A. Ladyzhenskaya und G. A. Seregin, On partial regularity of suitable weak solutions to the three-dimensional Navier-Stokes equations. J. Math. Fluid Mech. 1 (1999), 356-387.

[28] J. Leray, Sur le mouvement d'un liquide visqueux emplissant l'espace. Acta Math. 63 (1934), 193-248.
[29] F.H. Lin, A new proof of the Caffarelli-Kohn-Nirenberg theorem. Comm. Pure Appl. Math. LI (1998), 241-257.

[3o] J. Nečas, M. Růžička und V. Šverák, On Leray's self-similar solutions of the Navier-Stokes equations. Acta Math. 176 (1996), 283-294.

[31] C. W. Oseen, Neuere Methoden und Ergebnisse in der Hydrodynamik. Leipzig: Akademische Verlagsgesellschaft 1927.

[32] W.S. Ożański, Weak solutions to the Navier-Stokes inequality with arbitrary energy profiles. Comm. Math. Phys. 374 (2020), $33-62$.

[33] J. Serrin, The initial value problem für the Navier-Stokes equations. In Nonlinear Problems. Univ. Wisconsin Press (R. E. Langer, ed.), 2 69-98 (1963).

[34] H. Sohr, The Navier-Stokes Equations. An Elementary Functional Analytic Approach. Birkhäuser Advanced Texts. Birkhäuser Verlag Basel, Boston, Berlin 2001.

[35] V. A. Solonnikov, Estimates for solutions of nonstationary NavierStokes equations. Zap. Nauchn. Sem. LOMI 38 (1973), 153-231 (Russian).

[36] T. Tao, Finite time blowup for an averaged three-dimensional Navier-Stokes equation. J. Amer. Math. Soc. 29 (2016), 601-674.

[37] T.-P. Tsai, On Leray's self-similar solutions of the Navier-Stokes equations satisfying local energy estimates. Arch. Rational Mech. Anal. 143 (1998), 29-51. Erratum: Arch. Rational Mech. Anal. 147 (1999), 363.

[38] B. Wang, Ill-posedness for the Navier-Stokes equations in critical Besov spaces $\dot{B}_{\infty, q}^{-1}$. Adv. Math. 268 (2015), 350-372.

[39] J. Wolf, On the local regularity of suitable weak solutions to the generalized Navier-Stokes equations. Ann. Univ. Ferrara Sez. VII Sci. Mat. 61 (2015), 149-171.

[40] T. Yoneda, Ill-posedness of the $3 \mathrm{D}$-Navier-Stokes equations in a generalized Besov space near $B M O^{-1}$. J. Funct. Anal. 258 (2010), $337^{6}-3387$. 